\title{
Building with Ions in the Helium Ion Microscope
}

\author{
Olga S. Ovchinnikova ${ }^{1,2}$
}

${ }^{1 .}$ Center for Nanophase Materials Science, Oak Ridge National Laboratory, Oak Ridge, TN, USA

${ }^{2}$ The Institute for Functional Imaging of Materials and the Center for Nanophase Materials Sciences, Oak Ridge National Laboratory, Oak Ridge, TN, USA

In-situ direct writing by electron and ion beam from solutions opens a pathway for resistless fabrication of nanostructures at high throughput. This method utilizes the electron beam (e-beam) or ion beam (ibeam) as an ionizing source that decomposes solvent (specifically water here) molecules to generate solvated electrons and other radicals in a complex cascade of reaction pathways [1,2]. In water, radiolytically generated aqueous electrons $\left(\mathrm{e}_{\mathrm{aq}}{ }^{-}\right)$act as a reducing agent when interacting with organic and inorganic compounds, this may be harnessed to guide local deposition of the material directly from the precursor. Since electron and ion beam microscopes generally operate in high vacuum, (except variable pressure scanning electron microscope) it is necessary to utilize cells to encapsulate the liquid precursor in order to prevent evaporation [3,4]. These liquid cells are composed of silicon microchips and silicon nitride membranes [3,5]. In-situ liquid cell electron microscopy, has been used to investigate the mechanisms of electron beam induced nucleation, growth, and coalescence of nanoparticles in a variety of precursors at various concentrations [6-11]. To date, most in-situ liquid cell studies have been performed using a scanning electron microscope (SEM) or a (scanning) transmission electron microscope (S)TEM [12].

Use of the helium beam with the opposite charge and shorter mean free path offers the potential for the localization of the reaction zone on the single digit nanometer scale. Here we will present our results demonstrating writing of platinum structures from liquid in a platinum chloride solution using helium ions with sub-10 nm resolution (Figure 1). Using data analytics on acquired in-situ growth movies (Figure 2) we are able to elucidate the main statistical descriptors for helium ion beam initiated platinum structure growth. The possible mechanisms of beam induced growth and ultrahigh localization of reaction zone are discussed. Furthermore, we will discuss optimization of solution chemistry and instrumental parameters as they relate to the quality and thickness of structures and the extension to device fabrication on a single digit nanometer level [12].

\section{References:}

[1] Grogan, J. M. et al, Nano letters 14 (2013), p. 359.

[2] Schneider, N. M. et al, The Journal of Physical Chemistry C 118 (2014), p. 22373.

[3] de Jonge, N.; Ross, F. M., Nature Nanotechnology 6 (2011), p. 695.

[4] Ross, F. M., Science 350 (2015), aaa9886.

[5] Yuk, J. M. et al, Science 336 (2012), p. 61.

[6] Abellan, P. et al, Chemical Communications 50 (2014), p. 4873.

[7] Evans, J. E. et al, Nano letters 11 (2011), p. 2809.

[8] Jungjohann, K. et al, Nano letters 13 (2013), p. 2964.

[9] Woehl, T. J. et al, Acs Nano 6 (2012), p. 8599.

[10] Zheng, H. et al, Science 324 (2009), p. 1309.

[11] Ievlev, A. V. et al, Acs Nano (2015). 
[12] This work was conducted at the Center for Nanophase Materials Sciences, which is a Department of Energy (DOE) Office of Science User Facility
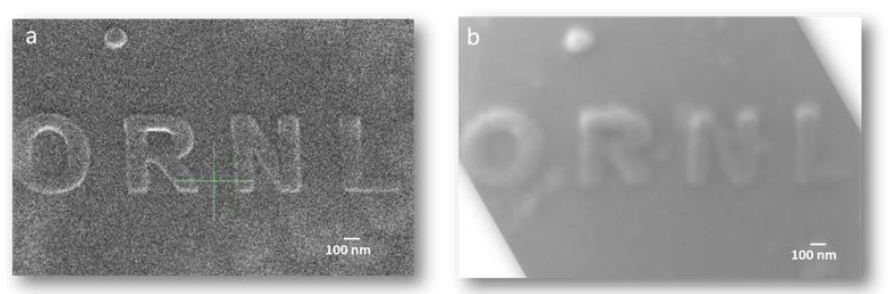

Figure 1. Imaging of deposited direct write platinum structure with a) in-situ in HIM and b) ex-situ via SEM

\section{Particle growth as a Function of Ion Dose}

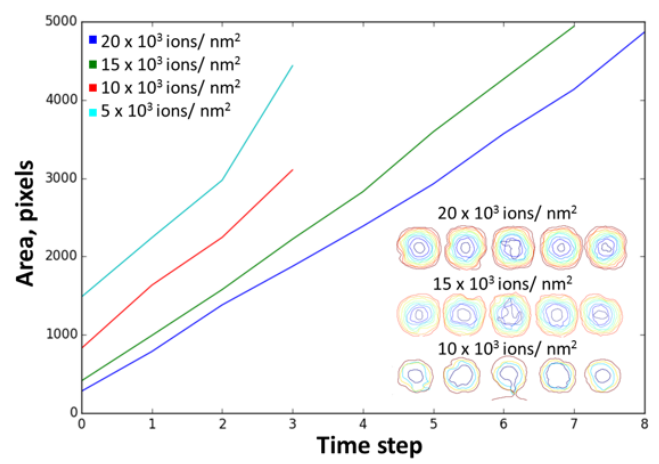

Figure 2. Data analytics of movie growth videos under different ion dose conditions reveals that the rate nucleation and growth of the Pt particles increases with decreased ion beam fluences. 